\title{
Confinedly Assembling Surface Nanocoating to Manipulate Nanofiltration Membranes for Highly-efficient Dye Removal
}

\author{
Xiaobin Yang, Fangjie You, Yuanyuan Zhao, Yongping Bai, and Lu Shao*
}

The increasing concern on water pollution and water-energy nexus have aroused the in-depth research on the eco-friendly design of molecule-separating nanofiltration membranes capable for both surface water and ground water treatments. Herein, a novel nanocoating was constructed by the layer-by-layer (LBL) assembly approach for confining the reaction between plant-derived polyphenol tannic acid (TA) and water-soluble polycation polyethyleneimine (PEI) to build advanced nanofiltration membrane focusing on dye removal in wastewater. Our approach can avoid the failure of the formation of nanofilm on porous membranes owing to instantaneous flocculation by the traditional direct co-deposition method. In addition, the entire assembling procedure was conducted in aqueous solution at ambient conditions. The incubation conditions and surface nanostructure and chemistry were investigated in detail for optimizing surface manipulation technology and improving membrane efficiency. PEI played significant roles in improving the membrane surface wettability and infiltration performance. The optimized membrane toward methyl blue (MB) and Victoria blue B (VB) removal gave the rejection rate beyond $92 \%$ with permeance of $c a .57 .6 \mathrm{~L} \mathrm{~m}^{-2} \mathrm{~h}^{-1} \mathrm{bar}^{-1}$. The delicate control of assembly conditions can flexibly regulate the membrane performance trade-off. The analogous polyphenol-based assemblies would power the advancement of membrane surface engineering for water treatments towards the bright water-energy nexus.

Keywords: Membrane separation; Nanofiltration; Dye removal; Layer-by-layer assembly; Water treatment

Received 20 August 2018, Accepted 12 September 2018

DOI: $10.30919 /$ esee 8 c142

\section{Introduction}

The water-energy nexus is receiving increasing concerns for the interdependence between water for energy production and energy for water treatment/production. ${ }^{1-5}$ Particularly, using energy-efficient molecular separation approaches for pollution remediation from overdischarged printing and textile wastewater are highly desirable. ${ }^{6-9}$ Different from conventional tackling methods, ${ }^{10-12}$ nanofiltration membrane exhibits extraordinary separation merits owing to the facile operation and non-phase transformation. It could validly fractionate solutes ranging from 200 to $1000 \mathrm{Da}$ which is well suitable for dye molecular removal from dyeing wastewater, desalination, and and antibiotics separation. ${ }^{13-17}$ However, overcoming the ubiquitous tradeoff between membrane permeability and selectivity are the key point to increase the energy efficiency of membrane processes. ${ }^{18-26}$ Therefore, the membrane surface manipulation has been considered as the useful tool to optimize the performance to overcome "trade-off" phenomenon for highly-efficient molecular separation.

Therein, from dedicated observation and research of the tightly adhered marine mussels on coastline rocks, Messersmith and his colleagues made a breakthrough in the field of surface engineering chemistry. They found that dopamine (DA) can simulate the

MIIT Key Laboratory of Critical Materials Technology for New Energy Conversion and Storage, State Key Laboratory of Urban Water Resource and Environment, School of Chemistry and Chemical Engineering, Harbin Institute of Technology, Harbin 150001, PR China

*E-mail : shaolu@hit.edu.cn chemical moieties of mussel's foot proteins and realize the robust and substrate-independent adhesion through self-polymerization into polydopamine (PDA) under aerobic alkaline conditions. ${ }^{27}$ Afterward, it has been widely accepted that the catechol, pyrogallol, and amino moieties greatly contribute to the benign adhesion of PDA and derived molecules, ${ }^{28-33}$ although further continuous endeavours are needed to uncover the exact adhesion mechanism therein. ${ }^{34-35}$ Inspired by the relationship between chemical structure and function of the mussel-inspired PDA, low-cost polyphenol compound system such as polyphenol/amine derivatives have been excavated for diverse usages such as surface manipulation. ${ }^{36-40}$ The plant-derived polyphenol tannic acid (TA) is the emerging cost-effective candidate for providing new surface manipulation platform. Meanwhile, polycations, such as polyethyleneimine (PEI), play a critical role in positive-charged membrane fabrication and charged molecules separation. ${ }^{41}$ However, the traditional polyphenol TA/polycation codeposition strategy will bring the instantaneous flocculation, resulting in the failure of formation of the coating layer on porous membranes with the molecular-selective function for nanofiltration applications. There exists the great challenge to realize the economical mussel-inspired coating for molecular separations towards the better water-energy nexus. Therein, the layer-by-layer (LBL) assembly strategy demonstrates the powerful advantage owing to the tunable surface chemistry and performance. ${ }^{42-46}$

Herein, we confined nanocoating formation at membrane surface via LBL assembly of TA/PEI incubation system to build mussel-inspired nanofiltration with manipulated performance. The entire assembling procedure was conducted in aqueous solution at 
ambient conditions with the aid of electrostatic attractions. By resorting to the control variable method, the physicochemical characterization and performance toward dyeing wastewater purification were systematically investigated on fabricated membranes via tuning TA and PEI respective concentrations only in one cycle. By optimizing the membrane infiltration trade-off, that effect of the assembly layer was further investigated. The performance demonstrated during TA/PEI assembly procedure exhibited that the LBL approach played a critical role in fabricating advanced nanofiltration membranes with superb water-purification performance.

\section{Experimental}

\subsection{Materials}

Tannic acid (TA), polyethyleneimine (PEI, $M_{W}=1800 \mathrm{~g} \mathrm{~mol}^{-1}$ ), methyl blue (MB, $M_{W}=799.80 \mathrm{~g} \mathrm{~mol}^{-1}$ ), and Victoria blue B (VB, $M_{W}=506.08 \mathrm{~g} \mathrm{~mol}^{-1}$ ) were purchased from Aladdin (China). Polymer (P84 polyimide: PI) for the support substrate was supplied by HP Polymer GmbH. The dyes were listed in Fig. S1. Ultrapure water was homemade.

\subsection{Membrane Preparations}

Pristine porous support membranes (polymer concentration, 15 wt.\%) were prepared according to our previous work. ${ }^{47}$ Then TA and PEI aqueous solutions with respective concentrations of $0.5,1,2$, and $5 \mathrm{mg} \mathrm{mL}^{-1}$ were prepared. The pristine membrane was immersed and gently shaken in TA and PEI aqueous solutions in sequence for $10 \mathrm{~s}$, respectively. A series of membranes with fixed PEI concentration of $5 \mathrm{mg} \mathrm{mL}^{-1}$ were prepared and membranes with fixed TA concentration of $1 \mathrm{mg} \mathrm{mL}^{-1}$ were further prepared. The membranes incubated by the TA and PEI concentrations at $m$ and $n$ $\mathrm{mg} \mathrm{mL}{ }^{-1}$, respectively, were denoted as TA/PEI $(m: n)$. In addition, to investigate the influence of varied assembled layers, the composite membranes composed of different assembled layers (membranes assembled with $x$ Layers were abbreviated as TA/PEI $(x \mathrm{~L})$ were fabricated while fixing the TA and PEI concentrations at 1 and $2 \mathrm{mg}$ $\mathrm{mL}^{-1}$, respectively. The membranes were rinsed with ultrapure water at the interval of every assembly cycle.

\subsection{Membrane Characterizations}

Attenuated total reflection flourier transformed infrared spectroscopy (ATR-FTIR) was carried out using a Spectrum One instrument (Perkin Elmer, USA). X-ray photoelectron spectroscopy (XPS) analysis was determined using Shimadzu AXIS Ultra DLD with an Al-K $\alpha$ X-ray source and the photoelectron take-off angle was $90^{\circ}$ in regard to the specimen surface. Scanning electron microscopy (SEM) images were collected from Hitachi S-4500. The specimens were fractured by immersing them in liquid nitrogen to observe the cross-sectional SEM images. Atomic force microscopy (AFM) images were measured by Solver P47 AFM (Russia) with the scanning area of $1 \times 1 \mu \mathrm{m}$. Water contact angles (WCA) were recorded by a SL $200 \mathrm{~KB}$ measure system. All membrane specimens were dried overnight under vacuum at $40^{\circ} \mathrm{C}$ prior to characterizations.

\subsection{Membrane Performance}

The performance (permeance and rejection) of membranes were conducted on a homemade dead-end nanofiltration equipment (Fig. S2). The membrane specimen was sealed by a rubber O-ring. The feed dye solutions (50 ppm) were poured into the measurer with valid membrane test area of $21.2 \mathrm{~cm}^{-2}$ and kept stirring at $800 \mathrm{rpm}$ for reducing the concentration of polarization under 5 bar derived from a nitrogen tank. The membranes were initially compacted for about one hour at 5 bar to obtain a steady status prior to the evaluation of separation performance. Permeance was calculated using Equation 1:

$$
P=\frac{V}{A \times t \times \Delta P}
$$

Where $P$ corresponds to the permeance $\left(\mathrm{L} \mathrm{m}^{-2} \mathrm{~h}^{-1} \mathrm{bar}^{-1}\right) . V, A, t$, and $\Delta P$ refer to the volume of permeate, effective membrane area, operation time, and transmembrane pressure, respectively.

Rejection was calculated using Eq. (2):

$$
R=\left(1-\frac{C_{p}}{C_{f}}\right) \times 100 \%
$$

Where $R$ corresponds to rejection. $C_{p}$ and $C_{f}$ refer to the concentration of dyes in permeate and feed, which were determined by a UV-vis Cintra20-GBC measurer.

\section{Results and discussion}

\subsection{Physicochemical characterizations of membranes}

The schematic of the eco-friendly LBL assembly of TA/PEI onto support membranes for dye removal in wastewater is presented in Fig. 1. The whole fabrication was eco-friendly and absent of any toxic solvent, fussy manipulation, and specific instruments. The TA/PEI LBL assembly could readily form on the porous substrate based on the benign biological adhesion of polyphenol TA molecules and electrostatic forces. The incubation time of porous membrane in respective solution just lasted for $10 \mathrm{~s}$ and it is a quite time-saving process.

ATR-FTIR spectra were utilized to detect the variation of functional moieties and mutual interactions between TA and PEI on membrane surface during confined assembly (Fig. 2). The corresponding FTIR spectra of pristine support membranes and membrane after only TA treatment were conducted as contrast. The new peaks located at 1716 and $1199 \mathrm{~cm}^{-1}$ corresponds to the $\mathrm{C}=\mathrm{O}$ and $\mathrm{C}-\mathrm{OH}$ stretching vibrations from TA molecules, respectively, when compared to that of support membrane. The components at 1615 and $1478 \mathrm{~cm}^{-1}$ referred to the characteristic peaks of nitrous carbons $(\mathrm{C}-\mathrm{N})$. The emerged spectrum at $1769 \mathrm{~cm}^{-1}$ of the TA/PEI LBL assembled membranes referred to the N-H stretching vibrations, which confirmed the immobilization of PEI molecules on the membrane surface with the aid of the robust adhesion of TA molecules. Meanwhile, the peak intensity at 1199 and $1478 \mathrm{~cm}^{-1}$ were weakened owing to the hydrogen bonds. In addition, the broader peaks at the location more than $3100 \mathrm{~cm}^{-1}$ confirmed the existence of hydrogen bonds in LBL assembling membranes, which were slightly different (peak shift) from the hydrogen bonds existing in the pristine support membrane, especially at higher TA contents (more -OH groups). The formation of TA/PEI coatings was further verified by the XPS analysis (Fig. S3). 


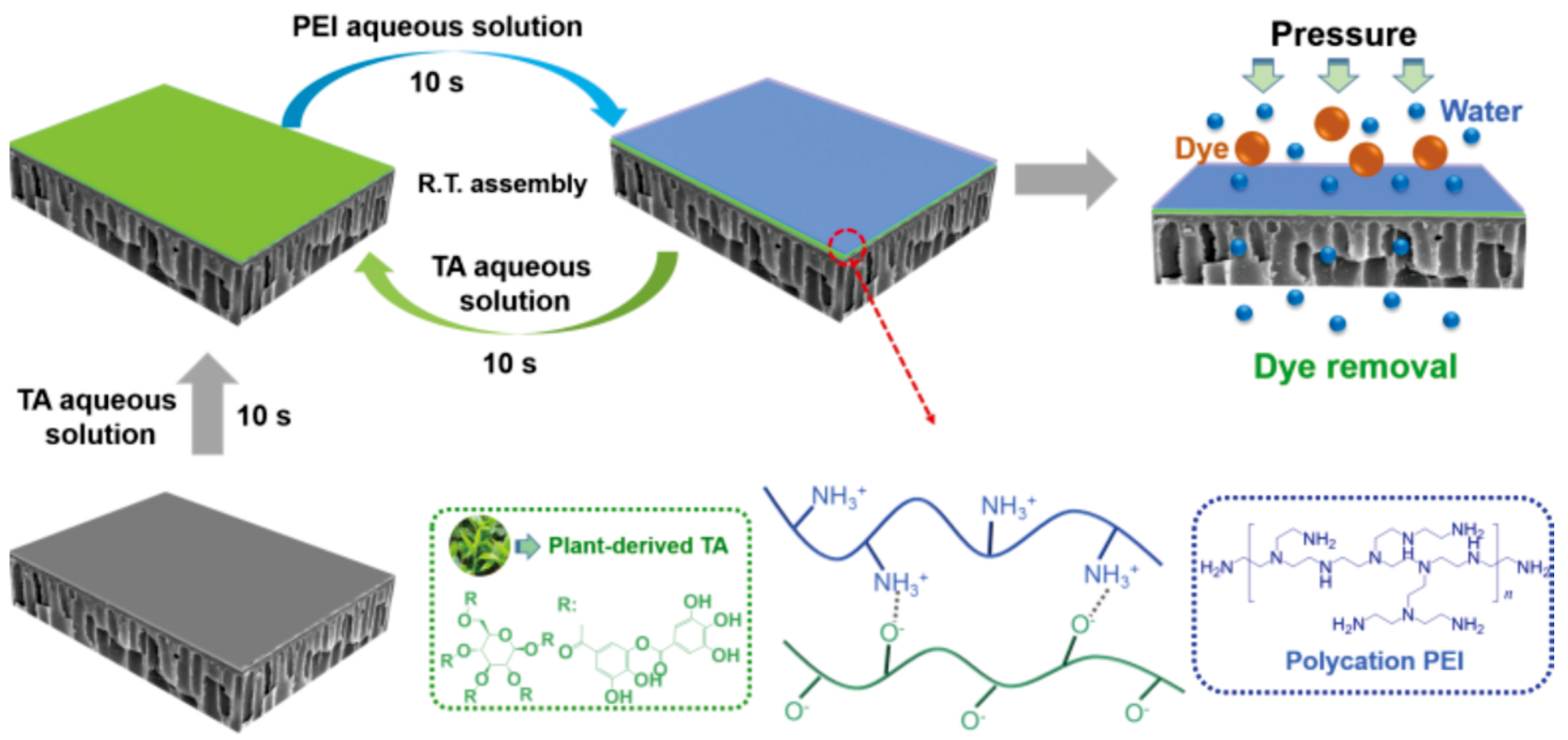

Porous substrate

Fig. 1 Schematic of the layer-by-layer assembly of TA/PEI onto porous substrate via confined reaction for dye removal. TA is the abbreviation of tannic acid, PEI is polyethyleneimine.

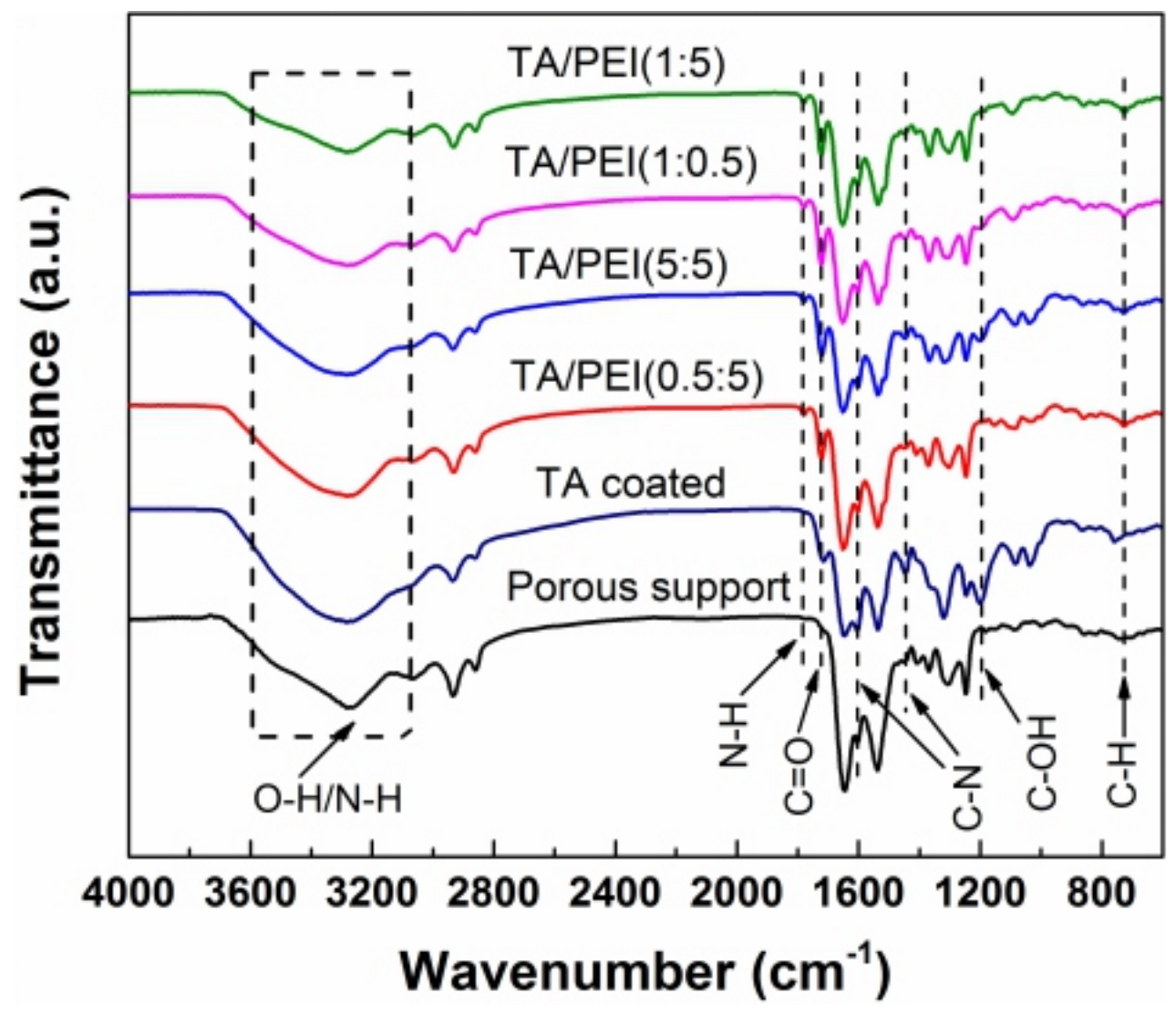

Fig. 2 ATR-FTIR spectra of pristine porous support, only TA coated membranes, and TA/PEI LBL assembled membranes with different TA/PEI mass ratios (the respective units of $\mathrm{m}: \mathrm{n}$ within the bracket are $\mathrm{mg} \mathrm{mL}^{-1}$ ). 
SEM and AFM were performed to clarify the relationship between membrane structure and LBL assembly conditions. As shown in Fig. 3, there are some observable aggregates distributed on the LBL assembling membrane surfaces, which are attributed to the strong hydrogen bonds and electrostatic interaction derived from ionization of the TA (negatively-charged) and PEI (positivelycharged) molecules. Although our LBL assembly approach can control the reaction between TA and PEI to a certain degree, the fast reaction between the two materials produce the aggregates inevitably. Particularly, the membrane surface morphology is closely related to the different concentrations of TA and PEI aqueous solution. When the PEI concentration is fixed at $5 \mathrm{mg} \mathrm{mL}^{-1}$, it was found that the aggregates on membrane surface increase with TA concentrations, which could be due to the more PEI molecules attachment on the membrane surface by the increased pre-adhered TA molecules. This is also consistent with the results of XPS. However, while fixing TA concentration, the increment of PEI concentration can result in less aggregation on the membrane surface. Thus, increasing the PEI concentration could be beneficial to the formation of much smooth and uniform assembling layer. Moreover, from the cross-sectional SEM, we could find that the TA/PEI assembly treatment did not bring appreciable alteration on the porous structure of the membranes (Fig. S4). Meanwhile, the thickness of the LBL assembling layer increased with both the TA and PEI concentration, which can affect membrane efficiency for separations.
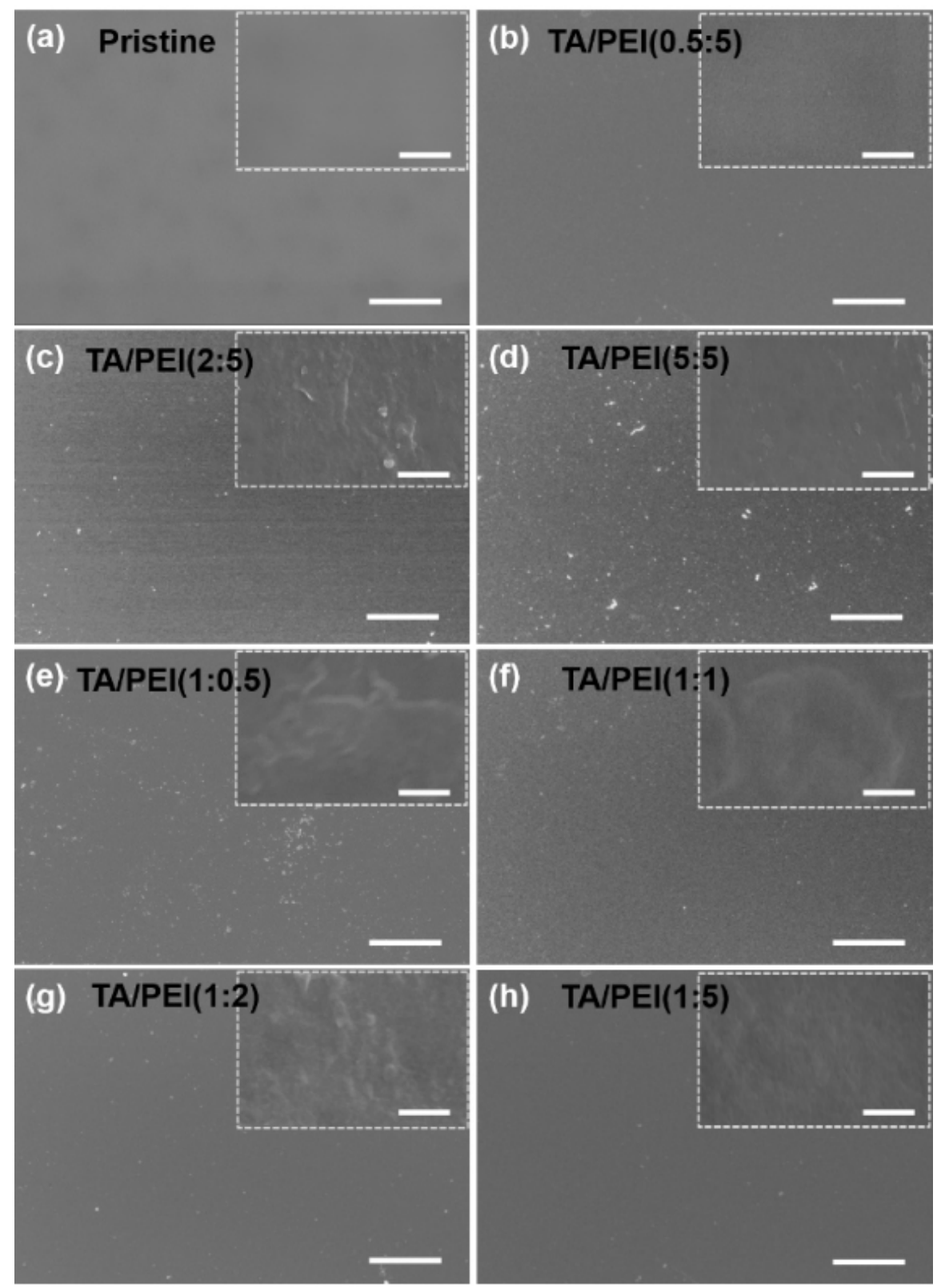

Fig. 3 SEM images of (a) pristine support, membranes assembled with respective TA concentrations of (b) 0.5 , (c) 2 , (d) $5 \mathrm{mg} \mathrm{mL}^{-1}$ while fixing PEI concentration at $5 \mathrm{mg} \mathrm{mL}^{-1}$, and membranes assembled with respective PEI concentrations of (e) 0.5 , (f) 1 , (g) 2 , (h) $5 \mathrm{mg} \mathrm{mL}^{-1}$ while fixing the TA concentration at $1 \mathrm{mg} \mathrm{mL}^{-1}$. The scale bars in large images are $20 \mu \mathrm{m}$. The scale bars in insets images are $500 \mathrm{~nm}$. 
Furthermore, the membrane surfaces were probed by AFM and the quantified average arithmetic roughness (Ra) was recorded (Fig. 4). The support membrane exhibited the typical smooth morphology $(\mathrm{Ra}=1.19 \mathrm{~nm})$. After TA/PEI assembly on support membrane, surface-bound TA/PEI nanocomplexes anchored at the membrane surface owing to the physicochemical interactions. The adhered TA molecules on the membrane surface with the aid of the adhesive pyrogallol moieties, tightly locked the PEI molecules, resulting in surface morphology evolution from the typical smooth surface of support membrane to the ridge-and-valley structural surface of the LBL assembling membranes. The AFM results are similar to that of SEM. The increased TA and PEI concentration resulted in enhanced $\mathrm{Ra}$ values of the membrane surfaces. However, the further improved aggregation density will result in a decrement in entire Ra value of membranes. The new hierarchical nanostructure has contributed to increase the membrane surface roughness as compared to pristine membrane, which can further enhance the surface wettability and infiltrating performance. ${ }^{18,21}$

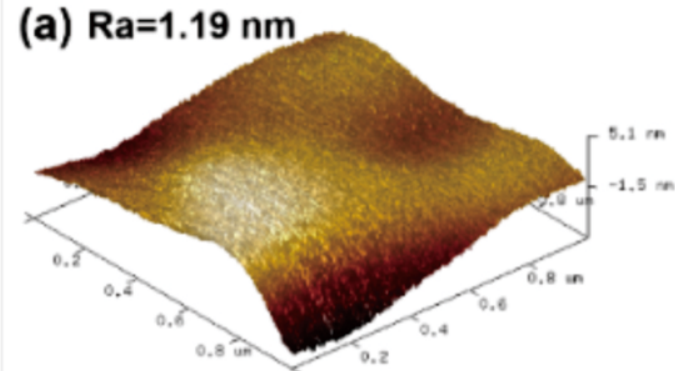

Pristine

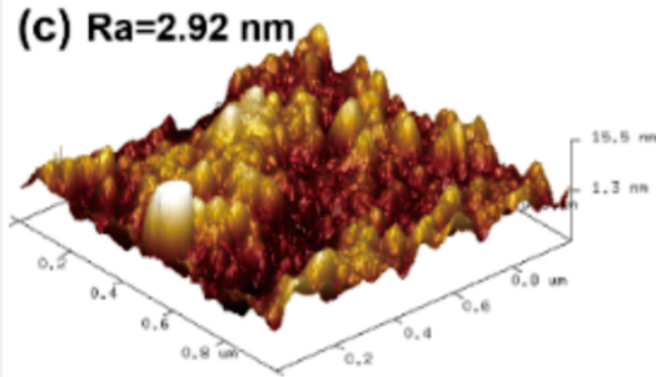

TA/PEI(2:5)

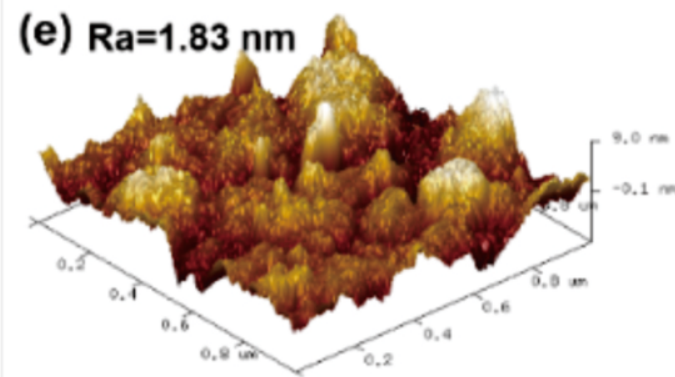

TA/PEI(1:0.5)

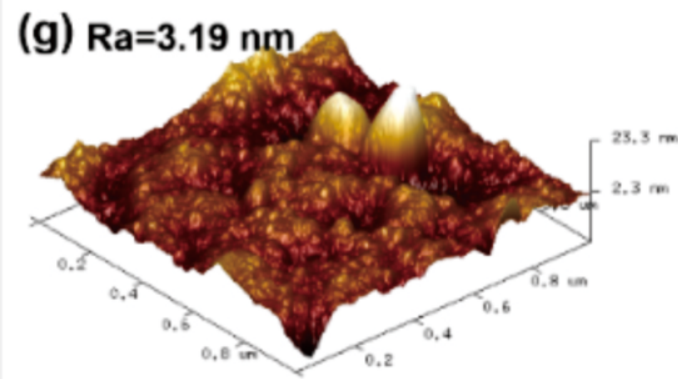

TA/PEI(1:2)

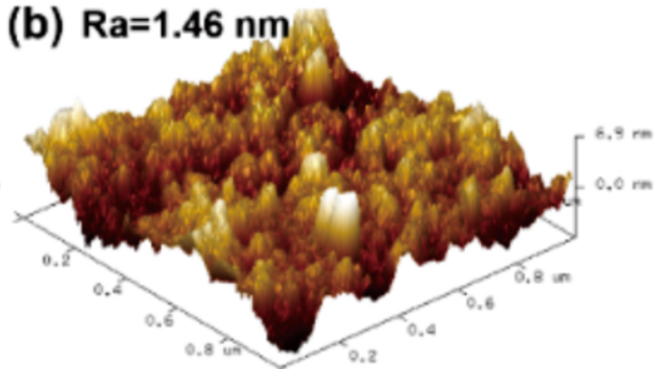

TA/PEI(0.5:5)

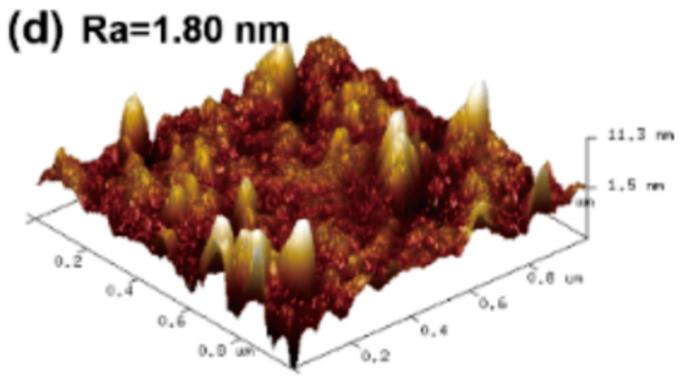

TA/PEI(5:5)

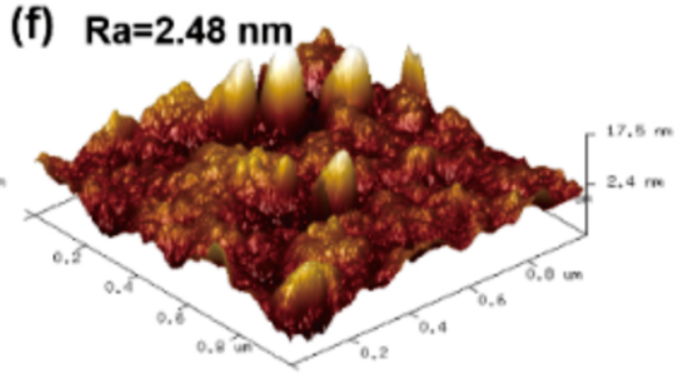

TA/PEI(1:1)

(h) $\mathrm{Ra}=1.98 \mathrm{~nm}$

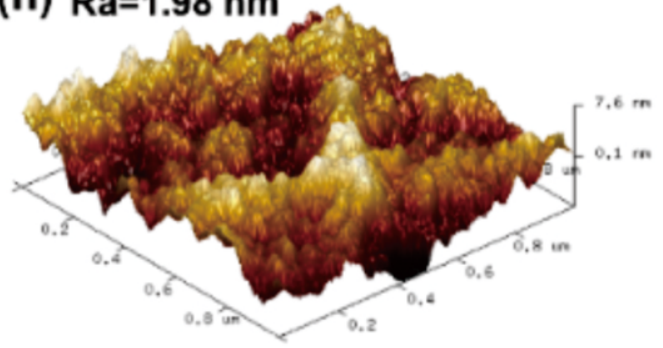

TA/PEI(1:5)

Fig. 4 The stereo AFM height modulation images of (a) pristine support and (b-h) TA/PEI LBL assembled membranes with different TA/PEI mass ratios. 


\subsection{Membrane wettability}

The ample exposed polar moieties, such as hydroxyl and amino groups, brought by the decorated TA/PEI assembly endowed the resultant membrane with enhanced wettability. ${ }^{48-50}$ As shown in Fig. 5 , the LBL assembly can greatly decrease the water contact angle of support membranes $\left(61.2^{\circ}\right)$. While fixing the PEI concentration, increasing TA concentrations brought the enhanced wettability of the membrane with the water contact angles from $22.5^{\circ}$ to $15.0^{\circ}$. The increased TA molecules attached to the membrane surface can hold more PEI molecules during PEI assembling process. Meanwhile, the resultant hierarchical nano-structure induced by the TA/PEI assembly can also enhance the membrane wettability. On the other hand, maintaining the TA concentration and increasing PEI concentration largely boosted the wettability of resultant membranes and the WCA decreased as low as $18.6^{\circ}$. Combined with the XPS analysis, the variation of PEI concentrations brought greater influence on the surface element compositions of the resultant membranes, and $\mathrm{N}$ element content significantly increased and $\mathrm{O} / \mathrm{N}$ ratio readily decreased with PEI concentrations. Such chemical structure evolution also leads to the enhanced hydrophilicity of the nanofiltration membranes, which greatly reduces the filtration resistance during the filtration toward the aqueous solutions owing to the enhanced affinity to water. This feature finally affects the membrane separation efficiency.
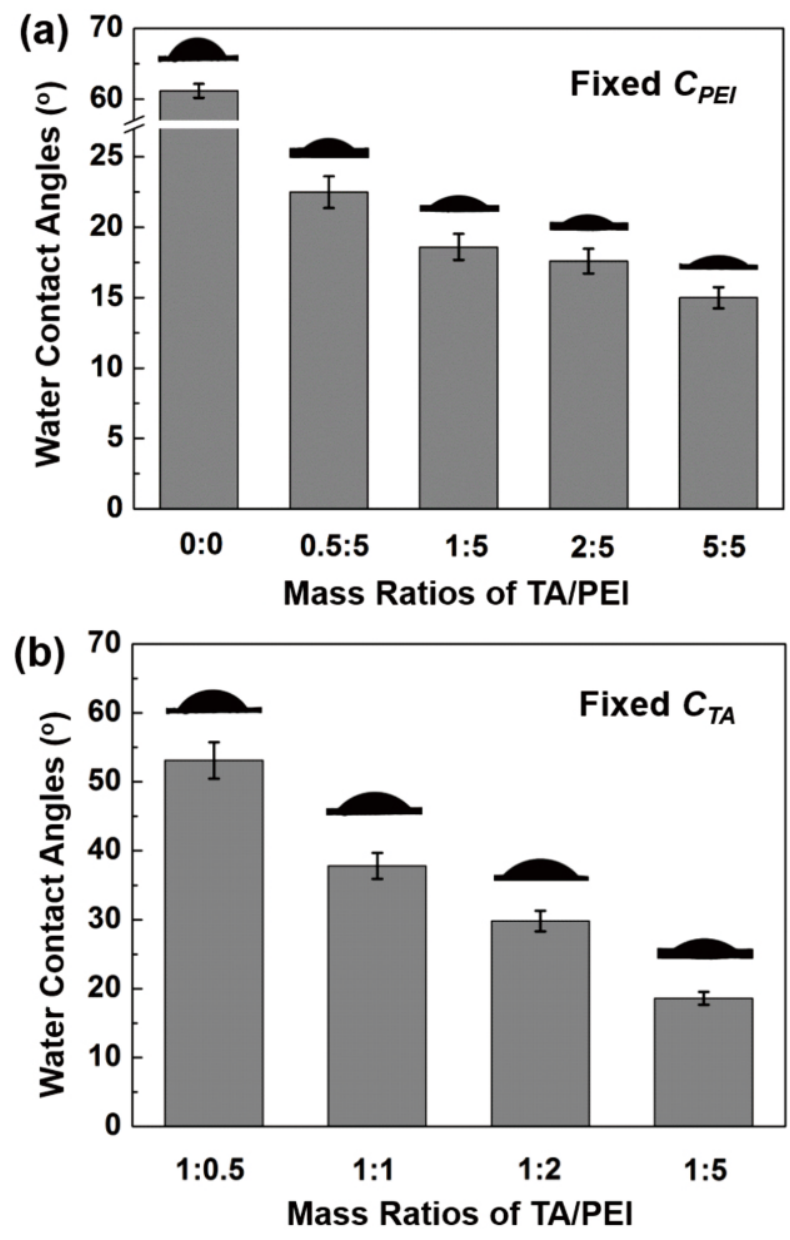

Fig. 5 Water contact angles of TA/PEI LBL assembled membranes fabricated by (a) fixed PEI concentration of $5 \mathrm{mg} \mathrm{mL}^{-1}$ (the sample for 0:0 is pristine membrane) and (b) fixed TA concentration of 1 $\mathrm{mg} \mathrm{mL}^{-1}$.

\subsection{Optimizing surface manipulation efficiency}

To improve the membrane efficiency towards water purification, the optimized concentrations of assembling materials were investigated based on one cycling assembly and the simulated dye wastewater. As shown in Fig. 6a, when the PEI concentration was fixed at $5 \mathrm{mg} \mathrm{ml}^{-1}$, increased TA concentrations during the assembly process resulted in a successive flux decline and rejection enhancement of the membranes. The permeance declined from $64.7 \mathrm{~L} \mathrm{~m}^{-2} \mathrm{~h}^{-1}$ bar $^{-1}$ to $3.4 \mathrm{~L} \mathrm{~m}^{-2} \mathrm{~h}^{-1}$ bar $^{-1}$ and the $\mathrm{MB}$ rejection increased from $85.5 \%$ to $99.8 \%$ and VB rejection increased from $88.2 \%$ to $100 \%$, respectively. Overall, combined with the aforementioned analysis, the improved intensity of anchored TA molecules induced the intensity of greater anchored PEI molecules, which introduced more compact selective layer on the membrane surface and resulted in decreased permeance flux and increased dye rejection. What's more, the modified membranes exhibited the better VB rejection than that of $\mathrm{MB}$, it is attributed to the Donnan effect occurred between the positively charged membrane surface brought by the externally anchored PEI and the dye solute with the same charge, VB.

In addition, the membrane efficiency for wastewater purification by varying the PEI concentrations was investigated (Fig. 6b). When TA concentration was fixed and PEI concentration
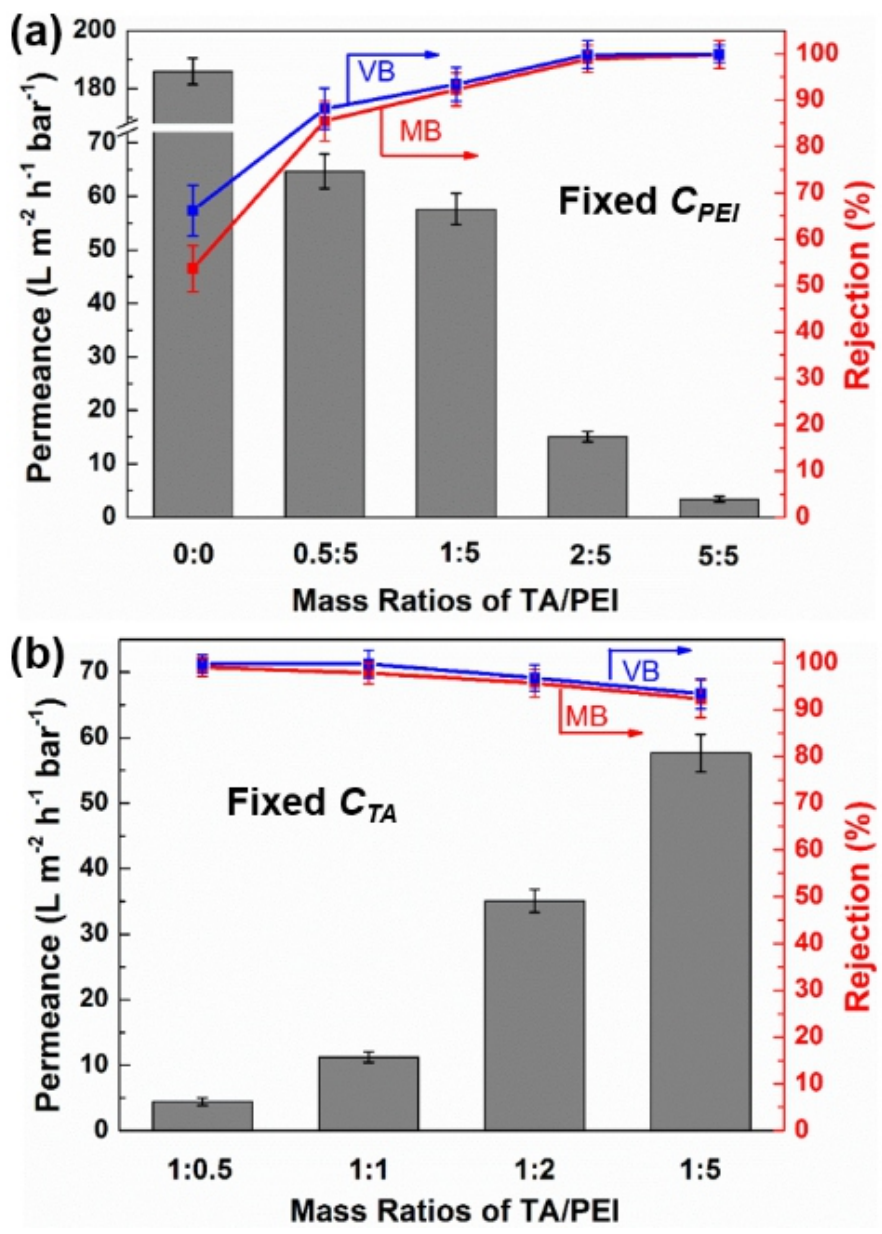

Fig. 6 Wastewater purification performance of membranes assembled with (a) the fixed PEI concentration of $5 \mathrm{mg} \mathrm{mL}^{-1}$ (the sample for 0:0 is pristine membrane) and (b) TA concentration of $1 \mathrm{mg} \mathrm{mL}$, respectively. 
was increased, the permeance significantly increased from $4.4 \mathrm{~L} \mathrm{~m}^{-2}$ $\mathrm{h}^{-1}$ bar $^{-1}$ to $57.6 \mathrm{~L} \mathrm{~m}^{-2} \mathrm{~h}^{-1}$ bar $^{-1}$. Different from the TA effect, the variation of PEI concentrations during the LBL assembling process greatly affected the wettability of the membranes, which could be confirmed by water contact angle results in Fig. 5. The wettability significantly increased with the PEI concentration which advanced the infiltrating performance and further enhanced the water permeance. Although the slight decline in the dye rejection is inevitable, the rejection for both dyes are still higher than $92 \%$ (92.3\% for MB and $93.4 \%$ for $\mathrm{VB})$. Therefore, the above results indicated the TA/PEI mass ratio of 1:2 during the assembly can produce much higher efficient membranes.

Furthermore, the effect of the TA/PEI assembly layers on the trade-off performance of membranes were researched under the TA/PEI mass ratio of 1:2 (Fig. 7). The assembled $n$ layers is denoted as $n \mathrm{~L}$. As for the specimen with 2 assembly layers, the permeance reduced from $35.1 \mathrm{~L} \mathrm{~m}^{-2} \mathrm{~h}^{-1}$ bar $^{-1}$ to $29.6 \mathrm{~L} \mathrm{~m}^{-2} \mathrm{~h}^{-1}$ bar $^{-1}$. With further increase of the assembly layers, the membrane permeation flux declined dramatically. It could be due to the increased assembly layer-induced the thicker selective layer, further leading to the increased mass transfer resistance during the separation process. When the assembled layer was up to 6 layers, the permeance of membrane was greatly reduced to $4.8 \mathrm{~L} \mathrm{~m}^{-2} \mathrm{~h}^{-1}$ bar $^{-1}$ although the MB rejection could increase from $95.7 \%$ to $99.8 \%$. The above results demonstrated the flexible controllability of the membrane trade-off between the permeance and rejection by varying the assembly layers. It indicated that there are enormous potential in delicate design and regulation of wastewater purification and performance of polyphenol-based LBL assembled membranes according to diverse demands.

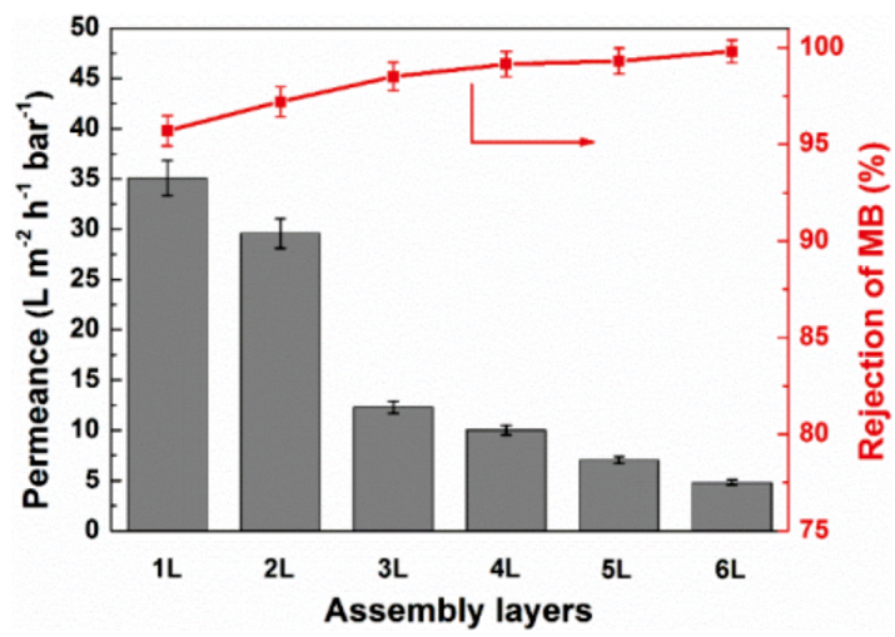

Fig. 7 Water purification performance of membranes with different assembly layers under the fixed TA/PEI mass ratio of 1:2.

\section{Conclusion}

Eco-friendly polyphenol/polyethyleneimine surface engineering was reported to construct high-performance nanofiltration membranes via LBL assembly towards highly-efficient membrane-based waterenergy nexus. According to the result, the assembling membranes exhibited better retention effect towards positively charged VB as compared with that of $\mathrm{MB}$ owing to the externally assembled positively charged PEI. The variation of PEI concentrations and microscopic ridge-and-valley texture could significantly affect the membrane surface wettability and further enhanced the filtrating performance; water contact angles decreased from $61.2^{\circ}$ to $18.6^{\circ}$. The optimized membrane toward MB and VB removal gave the rejection rate beyond $92 \%$ with permeance of $c$ a. $57.6 \mathrm{~L} \mathrm{~m}^{-2} \mathrm{~h}^{-1} \mathrm{bar}^{-1}$. Meanwhile, delicate control of assembly conditions and layers could further provide an enormous flexibility in design and regulation of the membrane filtrating trade-off according to the demands; it could realize the delicate control of permeance from $35.1 \mathrm{~L} \mathrm{~m}^{-2} \mathrm{~h}^{-1} \mathrm{bar}^{-1}$ to $4.8 \mathrm{~L} \mathrm{~m}^{-2} \mathrm{~h}^{-1}$ bar $^{-1}$ (MB rejection ranging from $95.7 \%$ to $99.8 \%$ ) by varying the TA/PEI assembly layers by adopting TA/PEI (1:2) as the target. Most importantly, polyphenol-based assemblies using the analogous functionalization approaches will promisingly accelerate eco-friendly membrane surface modification towards environmentfriendly and energy-saving usages.

\section{Conflicts of interest}

The authors declare no competing financial interest.

\section{Acknowledgments}

The work was financially supported from National Natural Science Foundation of China (21676063, U1462103), State Key Laboratory of Urban Water Resource and Environment (Harbin Institute Technology) (No. 2017DX07), and HIT Environment and Ecology Innovation Special Funds (HSCJ201619).

\section{Supplementary material}

The molecular structures of dyes, the schematic of the homemade dead-end nanofiltration measurer, XPS analysis and cross-sectional SEM images of membranes.

\section{References}

1. D. J. Miller, D.R. Dreyer, C.W. Bielawski, D.R. Paul, B.D. Freeman, Angew. Chem. Int. Ed., 2016, 56, 4662-4711.

2. S. Wang, K. Liu, X. Yao, L. Jiang, Chem. Rev., 2015, 115, 82308293.

3. J. W. Finley, J. N. Seiber, J. Agric. Food. Chem., 2014, 62, 62556262.

4. M. Elimelech, W. A. Phillip, Science, 2011, 333, 712-717.

5. H. B. Park, J. Kamcev, L. M. Robeson, M. Elimelech, B. D. Freeman, Science, 2017, 356, eaab0530.

6. B. Wang, W. Liang, Z. Guo, W. Liu, Chem. Soc. Rev., 2015, 44, 336-361.

7. P. Yang, Q. Liu, J. Liu, H. Zhang, Z. Li, R. Li, L. Liu, J. Wang, J. Mater. Chem. A, 2017, 5, 17933-17942.

8. Y.Q. Zhang, X.B. Yang, Z.X. Wang, J. Long, L. Shao, J. Mater. Chem. A, 2017, 5, 7316-7325.

9. M.A. Shannon, P.W. Bohn, M. Elimelech, J.G. Georgiadis, B.J. Mariñas, A.M. Mayes, Nature, 2008, 452, 301-310.

10. I. Ali, Chem. Rev., 2012, 112, 5073-5091.

11. A. G. Fane, R. Wang, M. X. Hu, Angew. Chem. Int. Ed., 2015, 54, 3368-3386.

12. R. Zhang, Y. Liu, M. He, Y. Su, X. Zhao, M. Elimelech, Z. Jiang, Chem. Soc. Rev., 2016, 45, 5888-5924.

13. X. Cheng, S. Ding, J. Guo, C. Zhang, Z. Guo, L. Shao, J. Membr. Sci., 2017, 536, 19-27.

14. P. Marchetti, M. F. J. Solomon, G. Szekely, A. G. Livingston, Chem. Rev., 2014, 114, 10735-10806. 
15. J. Wang, Y. Wang, Y. Zhang, A. Uliana, J. Zhu, J. Liu and d. B. B. Van, ACS Appl. Mater. Interfaces, 2016, 8, 25508-25519.

16. Y. Han, Y. Jiang and C. Gao, ACS Appl. Mater. Interfaces, 2015, 7, 8147-8155.

17. Y. C. Xu, Y. P. Tang, L. F. Liu, Z. H. Guo, L. Shao, J. Membr. Sci., 2017, 526, 32-42.

18. L.F. Villalobos, T. Huang, K.V. Peinemann, Adv. Mater, 2017, 29, 1606641.

19. J. Liu, Y. Xiao and T. S. Chung, J. Mater. Chem. A, 2017, 5, 4583-4595.

20. C. H. Lau, X. Mulet, K. Konstas, C. M. Doherty, M. A. Sani, F. Separovic, M. R. Hill and C. D. Wood, Angew. Chem. Int. Ed., 2016, 55, 1998-2001.

21. S. Karan, Z. Jiang, A. G. Livingston, Science, 2015, 348, $1347-$ 1351.

22. S. Ling, Q. Zhao, W. Huang, S. Cao, D. L. Kaplan, M. J. Buehler, Sci. Adv., 2017, 3, e1601939.

23. E. Barankova, X. Tan, L. F. Villalobos, E. Litwiller, K. V. Peinemann, Angew. Chem. Int. Ed., 2017, 56, 2965-2968.

24. H. Ang, L. Hong, ACS Appl. Mater. Interfaces, 2017, 9, 2807928088.

25. S. Hermans, E. Dom, H. Mariën, G. Koeckelberghs, I. F. J. Vankelecom, J. Membr. Sci., 2015, 476, 356-363.

26. C. Zhang, B. Cao, P. Li, J. Membr. Sci., 2018, 546, 90-99.

27. H. Lee, S. M. Dellatore, W. M. Miller, P. B. Messersmith, Science, 2007, 318, 426-430.

28. Z. Wang, X. Jiang, X. Cheng, C. H. Lau, L. Shao, ACS Appl. Mater. Interfaces, 2015, 7, 9534-9545.

29. M. A. Rahim, M. Björnmalm, N. Bertleff-Zieschang, Q. Besford, S. Mettu, T. Suma, M. Faria, F. Caruso, Adv. Mater. 2017, 29, 1606717.

30. T. S. Sileika, D.G. Barrett, R. Zhang, K. H. A. Lau, P.B. Messersmith, Angew. Chem. Int. Ed., 2013, 52, 10766-10770.

31. H. C. Yang, J. Hou, V. Chen and Z. K. Xu, J. Mater. Chem. A, 2016, 4, 9716-9729.
32. Z. Wang, X. Yang, Z. Cheng, Y. Liu, L. Shao, L. Jiang, Mater. Horiz., 2017, 4, 701-708.

33. Q. Wei, R. Haag, Mater. Horiz., 2015, 2, 567-577.

34. Y. Liu, K. Ai, L. Lu, Chem. Rev., 2014, 114, 5057-5115.

35. H. C. Yang, J. Luo, Y. Lv, P. Shen, Z. K. Xu, J. Membr. Sci., 2015, 483, 42-59.

36. F. Behboodi-Sadabad, H. Zhang, V. Trouillet, A. Welle, N. Plumeré, P. A. Levkin, Adv. Funct. Mater., 2017, 27, 1700127.

37. D. Pranantyo, L. Q. Xu, K. G. Neoh, E. T. Kang, Y. X. Ng, S.L. Teo, Biomacromolecules, 2015, 16, 723-732.

38. H. Ejima, F. Caruso, Science, 2013, 341, 154-157.

39. X. Yang, H. Sun, A. Pal, Y. Bai, L. Shao, ACS Appl. Mater. Interfaces, 2018, 10, 29982-29991.

40. X. Yang, Z. Wang, L. Shao. J. Membr. Sci., 2017, 5, 7316-7325.

41. G. Han, T.S. Chung, M. Weber, C. Maletzko, Environ. Sci. Technol., 2018, 52, 3676-3684.

42. F. Fan, C. Zhou, X. Wang, J. A. Szpunar, ACS Appl. Mater. Interfaces, 2015, 7, 27271-27278.

43. J. Zhao, F. Pan, P. Li, C. Zhao, Z. Jiang, P. Zhang, X. Cao, ACS Appl. Mater. Interfaces, 2013, 5, 13275-13283.

44. Q. C. Xia, J. Wang, X. Wang, B. Z. Chen, J. L. Guo, T. Z. Jia, S. P. Sun, J. Membr. Sci., 2017, 539, 392-402.

45. Y. Zhang, S. Zhang, J. Gao, T. S. Chung, J. Membr. Sci. 2016, 515, 230-237.

46. V. Kozlovskaya, E. Kharlampieva, I. Drachuk, D. Cheng, V. Tsukruk, Soft Matter, 2010, 6, 3596-3608.

47. F. You, Y. Xu, X. Yang, Y. Zhang, L. Shao, Chem. Commun., 2017, 53, 6128-6131.

48. X. Yang, X. Jiang, Y. Huang, Z. Guo, L. Shao, ACS Appl. Mater. Interfaces, 2017, 9, 5590-5599.

49. J. Zhu, L. Qin, A. A. Uliana, J. Hou, W. Jing, Y. Zhang, L. Xin, S. Yuan, L. Jian, M. Tian, ACS Appl. Mater. Interfaces, 2017, 9, 1975-1986.

50. X. Yang, H. Du, S. Li, Z. Wang, L. Shao, ACS Sustainable Chem. Eng., 2018, 6, 4412-4420. 